\title{
A DOUBLY CRITICAL SEMILINEAR HEAT EQUATION IN THE $L^{1}$ SPACE
}

\author{
YASUHITO MIYAMOTO
}

\begin{abstract}
We study the existence and nonexistence of a Cauchy problem of the semilinear heat equation

$$
\begin{cases}\partial_{t} u=\Delta u+|u|^{p-1} u & \text { in } \mathbb{R}^{N} \times(0, T), \\ u(x, 0)=\phi(x) & \text { in } \mathbb{R}^{N}\end{cases}
$$

in $L^{1}\left(\mathbb{R}^{N}\right)$. Here, $N \geq 1, p=1+2 / N$ and $\phi \in L^{1}\left(\mathbb{R}^{N}\right)$ is a possibly sign-changing initial function. Since $N(p-1) / 2=1$, the $L^{1}$ space is scale critical and this problem is known as a doubly critical case. It is known that a solution does not necessarily exist for every $\phi \in L^{1}\left(\mathbb{R}^{N}\right)$. Let $X_{q}:=\left\{\phi \in L_{\text {loc }}^{1}\left(\mathbb{R}^{N}\right)\left|\int_{\mathbb{R}^{N}}\right| \phi \mid[\log (e+|\phi|)]^{q} d x<\infty\right\}\left(\subset L^{1}\left(\mathbb{R}^{N}\right)\right)$. In this paper we construct a local-in-time mild solution in $L^{1}\left(\mathbb{R}^{N}\right)$ for $\phi \in X_{q}$ if $q \geq N / 2$. We show that, for each $0 \leq q<N / 2$, there is a nonnegative initial function $\phi_{0} \in X_{q}$ such that the problem has no nonnegative solution, using a necessary condition given by BarasPierre [Ann. Inst. H. Poincaré Anal. Non Linéaire 2 (1985), 185-212]. Since $X_{q} \subset X_{N / 2}$ $(q \geq N / 2), X_{N / 2}$ becomes a sharp integrability condition. We also prove a uniqueness in a certain set of functions which guarantees the uniqueness of the solution constructed by our method.
\end{abstract}

\section{INTRODUCTION AND MAIN RESULTS}

We consider the existence and nonexistence of a Cauchy problem of the semilinear heat equation

$$
\begin{cases}\partial_{t} u=\Delta u+|u|^{p-1} u & \text { in } \mathbb{R}^{N} \times(0, T), \\ u(x, 0)=\phi(x) & \text { in } \mathbb{R}^{N},\end{cases}
$$

where $N \geq 1, p=1+2 / N$ and $\phi$ is a possibly sign-changing initial function. When $\phi \in L^{\infty}\left(\mathbb{R}^{N}\right)$, one can easily construct a solution by using a fixed point argument. When $\phi \notin L^{\infty}\left(\mathbb{R}^{N}\right)$, the solvability depends on the balance between the strength of the singularity of $\phi$ and the growth rate of the nonlinearity. Weissler [13] studied the solvability of (1.1), and obtained the following:

Proposition 1.1. Let $q_{c}:=N(p-1) / 2$. Then the following (i) and (ii) hold:

(i) (Existence, subcritical and critical cases) Assume either both $q>q_{c}$ and $q \geq 1$ or $q=q_{c}>$ 1. The problem (1.1) has a local-in-time solution for $\phi \in L^{q}\left(\mathbb{R}^{N}\right)$.

(ii)(Nonexistence, supercritical case) For each $1 \leq q<q_{c}$, there is $\phi \in L^{q}\left(\mathbb{R}^{N}\right)$ such that (1.1) has no local-in-time nonnegative solution.

Date: December 25, 2019.

2010 Mathematics Subject Classification. Primary: 35K55; Secondary: 35A01, 46E30.

Key words and phrases. Local-in-time solution, Fujita exponent, Supersolution, Optimal singularity. 


\begin{tabular}{|c|c|c|c|c|}
\hline ranges of $q$ & $\begin{array}{c}1 \leq q<q_{c} \\
\text { supercritical }\end{array}$ & $\begin{array}{c}1=q=q_{c} \\
\text { doubly critical }\end{array}$ & $\begin{array}{c}1<q=q_{c} \\
\text { critical }\end{array}$ & $\begin{array}{c}q>q_{c}, q \geq 1 \\
\text { subcritical }\end{array}$ \\
\hline \multirow[t]{2}{*}{$\begin{array}{c}\text { existence/ } \\
\text { nonexistence }\end{array}$} & $\begin{array}{l}\text { not always } \\
\text { exist }\end{array}$ & $\begin{array}{c}\text { not always } \\
\text { exist }\end{array}$ & exist & exist \\
\hline & Prop. 1.1 (ii) & $\begin{array}{r}\text { exist: [14, p.32], } \\
\text { Thm. [1.3 (i) } \\
\text { not exist: [2, 3. [7], } \\
\text { Thm. [1.3 (ii) }\end{array}$ & Prop. 1.1 (i) & Prop. 1.1 (i) \\
\hline
\end{tabular}

TABLE 1. Existence and nonexistence of a local-in-time solution of (1.1) in $L^{q}\left(\mathbb{R}^{N}\right)$.

Let $u(x, t)$ be a function such that $u$ satisfies the equation in (1.1). We consider the scaled function $u_{\lambda}(x, t):=\lambda^{2 /(p-1)} u\left(\lambda x, \lambda^{2} t\right)$. Then, $u_{\lambda}$ also satisfies the same equation. We can easily see that $\left\|u_{\lambda}(x, 0)\right\|_{q}=\|u(x, 0)\|_{q}$ if and only if $q=q_{c}$. It is well known that $q_{c}$ is a threshold as Proposition 1.1 shows. However, the case $q=q_{c}=1$, i.e., $p=1+2 / N$, is not covered by Proposition 1.1, and it is known that there is a nonnegative initial function $\phi \in L^{1}\left(\mathbb{R}^{N}\right)$ such that (1.1) with $p=1+2 / N$ has no local-in-time nonnegative solution. See Brezis-Cazenave [2, Theorem 11], Celik-Zhou [3, Theorem 4.1] or Laister et.al. [7, Corollary 4.5] for nonexistence results. See [1, 6, 11 and references therein for existence and nonexistence results with measures as initial data. In [2, Section 7.5] the case $p=1+2 / N$ is referred to as "doubly critical case". Several open problems were given in [2]. It was mentioned in [14, p.32] that (1.1) has a local-in-time solution if $\phi \in L^{1}\left(\mathbb{R}^{N}\right) \cap L^{q}\left(\mathbb{R}^{N}\right)$ for some $q>1$. However, a solvability condition was not well studied. See Table 1. For a detailed history about the existence, nonexistence and uniqueness of (1.1), see [3], Section 1].

In this paper we obtain a sharp integrability condition on $\phi \in L^{1}\left(\mathbb{R}^{N}\right)$ which determines the existence and nonexistence of a local-in-time solution in the case $p=1+2 / N$. We also show that a solution constructed in Theorem 1.3 is unique in a certain set of functions. Throughout the present paper we define $f(u):=|u|^{p-1} u$. Let $L^{q}\left(\mathbb{R}^{N}\right), 1 \leq q \leq \infty$, denote the usual Lebesgue space on $\mathbb{R}^{N}$ equipped with the norm $\|\cdot\|_{q}$. For $\phi \in L^{1}\left(\mathbb{R}^{N}\right)$, we define

$$
S(t)[\phi](x):=\int_{\mathbb{R}^{N}} G_{t}(x-y) \phi(y) d y,
$$

where $G_{t}(x-y):=(4 \pi t)^{-N / 2} \exp \left(-\frac{|x-y|^{2}}{4 t}\right)$. The function $S(t)[\phi]$ is a solution of the linear heat equation with initial function $\phi$. We give a definition of a solution of (1.1).

Definition 1.2. Let $u$ and $\bar{u}$ be measurable functions on $\mathbb{R}^{N} \times(0, T)$.

(i)(Integral solution) We call $u$ an integral solution of (1.1) if there is $T>0$ such that $u$ satisfies the integral equation

$$
u(t)=\mathcal{F}[u](t) \text { a.e. } x \in \mathbb{R}^{N}, \quad 0<t<T, \quad \text { and }\|u(t)\|_{\infty}<\infty \text { for } 0<t<T,
$$

where

$$
\mathcal{F}[u](t):=S(t) \phi+\int_{0}^{t} S(t-s) f(u(s)) d s .
$$

(ii) (Mild solution) We call $u$ a mild solution if $u$ is an integral solution and $u(t) \in C\left([0, T), L^{1}\left(\mathbb{R}^{N}\right)\right)$. (iii) We call $\bar{u}$ a supersolution of (1.1) if $\bar{u}$ satisfies the integral inequality $\mathcal{F}[\bar{u}](t) \leq \bar{u}(t)<\infty$ for a.e. $x \in \mathbb{R}^{N}, 0<t<T$. 
For $0 \leq q<\infty$, we define a set of functions by

$$
X_{q}:=\left\{\phi(x) \in L_{\mathrm{loc}}^{1}\left(\mathbb{R}^{N}\right)\left|\int_{\mathbb{R}^{N}}\right| \phi \mid[\log (e+|\phi|)]^{q} d x<\infty\right\} .
$$

It is clear that $X_{q} \subset L^{1}\left(\mathbb{R}^{N}\right)$ and that $X_{q_{1}} \subset X_{q_{2}}$ if $q_{1} \geq q_{2}$. The main theorem of the paper is the following:

Theorem 1.3. Let $N \geq 1$ and $p=1+2 / N$. Then the following (i) and (ii) hold:

(i)(Existence) If $\phi \in X_{q}$ for some $q \geq N / 2$, then (1.1) has a local-in-time mild solution $u(t)$, and this mild solution satisfies the following:

$$
\text { there is } C>0 \text { such that }\|u(t)\|_{\infty} \leq C t^{-\frac{N}{2}}(-\log t)^{-q} \text { for small } t>0 .
$$

In particular, (1.1) has a local-in-time mild solution for every $\phi \in X_{N / 2}$.

(ii)(Nonexistence) For each $0 \leq q<N / 2$, there is a nonnegative initial function $\phi_{0} \in X_{q}$, which is explicitly given by (4.1), such that (1.1) has no local-in-time nonnegative integral solution, and hence (1.1) has no local-in-time nonnegative mild solution.

Remark 1.4. (i) The function $\phi$ in Theorem 1.3 (i) is not necessarily nonnegative.

(ii) Theorem 1.3 indicates that $X_{N / 2}\left(\subset L^{1}\left(\mathbb{R}^{N}\right)\right)$ is an optimal set of initial functions for the case $p=1+2 / N$, and $X_{N / 2}$ is slightly smaller than $L^{1}\left(\mathbb{R}^{N}\right)$. This situation is different from the case $p>1+2 / N$, since (1.1) is always solvable in the scale critical space $L^{N(p-1) / 2}$ for $p>1+2 / N$ (Proposition 1.1 (i)).

(iii) $L^{1}\left(\mathbb{R}^{N}\right)$ is larger than the optimal set for $p=1+2 / N$. On the other hand, it follows from Proposition 1.1 (i) that if $1<p<1+2 / N$, then (1.1) has a solution for all $\phi \in L^{1}\left(\mathbb{R}^{N}\right)$. Therefore, $L^{1}\left(\mathbb{R}^{N}\right)$ is small enough for the case $1<p<1+2 / N$.

(iv) The function $\phi_{0}$ given in Theorem 1.3 (ii) is modified from $\psi(x)$ given by (1.9). This function comes from Baras-Pierre [1], and Theorem 1.3 (ii) is a rather easy consequence of [1, Proposition 3.2]. However, we include Theorem 1.3 (ii) for a complete description of the borderline property of $X_{N / 2}$.

(v) Laister et.al. [7] obtained a necessary and sufficient condition for the existence of a localin-time nonnegative solution of

$$
\begin{cases}\partial_{t} u=\Delta u+h(u) & \text { in } \mathbb{R}^{N} \times(0, T) \\ u(x, 0)=\phi(x) \geq 0 & \text { in } \mathbb{R}^{N}\end{cases}
$$

They showed that when $h(u)=u^{1+2 / N}[\log (e+u)]^{-r}$, 1.4) has a local-in-time nonnegative solution for every nonnegative $\phi \in L^{1}\left(\mathbb{R}^{N}\right)$ if $1<r<\lambda p$, and (1.4) does not always have if $0 \leq r \leq 1$. Here, $\lambda>0$ is a certain constant. Therefore, the optimal growth of $h(u)$ for $L^{1}\left(\mathbb{R}^{N}\right)$ is slightly smaller than $u^{1+2 / N}$.

(vi) The exponent $p=1+2 / N$, which is called Fujita exponent, also plays a key role in the study of global-in-time solutions. If $1<p \leq 1+2 / N$, then every nontrivial nonnegative solution of (1.1) blows up in a finite time. If $p>1+2 / N$, then (1.1) has a global-in-time nonnegative solution. See Fujita [4]. In particular, in the case $p=1+2 / N$ we cannot expect a global existence of a classical solution for small initial data.

The next theorem is about the uniqueness of the integral solution in a certain class. 
Theorem 1.5. Let $N \geq 1, p=1+2 / N$ and $q>N / 2$. Then an integral solution $u(t)$ of (1.1) is unique in the set

$$
\left\{u(t) \in L^{1}\left(\mathbb{R}^{N}\right) \mid \sup _{0 \leq t \leq T} t^{N / 2}(-\log t)^{q}\|u(t)\|_{\infty}<\infty\right\} .
$$

Therefore, a solution given by Theorem 1.3 is unique.

Remark 1.6. (i) If there were a solution that does not satisfy (1.5), then the uniqueness fails. However, it seems to be an open problem.

(ii) In the case $q=N / 2$ the uniqueness under (1.5) is left open.

(iii) For general $p$ and $q$, the uniqueness of a solution of (1.1) is known in the set

$$
\left\{u(t) \in L^{q}\left(\mathbb{R}^{N}\right) \mid \sup _{0 \leq t \leq T} t^{\frac{N}{2}\left(\frac{1}{q}-\frac{1}{p q}\right)}\|u(t)\|_{p q}<\infty\right\} .
$$

See Haraux-Weissler [5] and [13]. For an unconditional uniqueness with a certain range of $p$ and $q$, see [2, Theorem 4].

(iv) The nonuniqueness in $L^{q}\left(\mathbb{R}^{N}\right)$ is also known for (1.1). For $p>1+2 / N$ and $1 \leq q<$ $N(p-1) / 2<p+1$, see [5]. For $p=q=N /(N-2)$, see $N i$-Sacks [8] and Terraneo [12].

Let us mention technical details. We assume that $\phi \in X_{q}$ for some $q \geq N / 2$. Using a monotone method, we construct a nonnegative mild solution $w(t)$ of

$$
\begin{cases}\partial_{t} w=\Delta w+f(w) & \text { in } \mathbb{R}^{N} \times(0, T), \\ w(x, 0)=|\phi(x)| & \text { in } \mathbb{R}^{N}\end{cases}
$$

We define $g(u)$ by

$$
g(u):=u[\log (\rho+|u|)]^{q},
$$

where $\rho>1$ is chosen appropriately. We will see that if $\rho \geq e$, then $g(u)$ is convex for $u \geq 0$ and $g$ plays a crucial role in the construction of the solution of (1.6). In order to construct a nonnegative solution we use a method developed by Robinson-Sierżȩga [10] with the convex function $g$, which was also used in Hisa-Ishige [6]. We define a sequence of functions $\left(u_{n}\right)_{n=0}^{\infty}$ by

$$
\left\{\begin{array}{l}
u_{n}(t)=\mathcal{F}\left[u_{n-1}\right](t) \text { for } 0 \leq t<T \text { if } n \geq 1 \\
u_{0}(t)=0
\end{array}\right.
$$

Then, we show that $-w(t) \leq u_{n}(t) \leq w(t)$ for $0 \leq t<T$. Since $\left|u_{n}(t)\right| \leq w(t)$, we can extract a convergent subsequence in $C_{\text {loc }}\left(\mathbb{R}^{N} \times(0, T)\right)$, using a parabolic regularization, the dominated convergence theorem and a diagonal argument. The limit function becomes a mild solution (1.1).

In the nonexistence part we use a necessary condition for the existence of a nonnegative solution of (1.1) obtained by Baras-Pierre [1], which is stated in Proposition 2.2 in the present paper. Using their result, one can show that there is $c_{0}>0$ such that if $\phi(x) \geq c_{0} \psi(x)$ in a neighborhood of the origin, then (1.1) has no nonnegative integral solution. Here,

$$
\psi(x):=|x|^{-N}(-\log |x|)^{-\frac{N}{2}-1} \text { for } 0<|x|<1 / e .
$$

See also [6]. For each $0 \leq q<N / 2$ we will see that a modified function $\phi_{0}$, which is given by (4.1), belongs to $X_{q}$. We show that $\phi_{0}$ does not satisfy the necessary condition for the 
existence of an integral solution stated in Proposition 2.2. Hence, (1.1) with $\phi_{0}$ has no nonnegative solution for each $0 \leq q<N / 2$.

This paper consists of five sections. In Section 2 we recall known results including a monotone method, a necessary condition on the existence for (1.1) and $L^{p}$ - $L^{q}$-estimates. In Section 3 we prove Theorem 1.3 (i). In Section 4 we prove Theorem 1.3 (ii). In Section 5 we prove Theorem 1.5.

\section{Preliminaries}

First we recall the monotonicity method.

Lemma 2.1. Let $0<T \leq \infty$ and let $f$ be a continuous nondecreasing function such that $f(0) \geq 0$. The problem (1.1) has a nonnegative integral solution for $0<t<T$ if and only if (1.1) has a nonnegative supersolution for $0<t<T$. Moreover, if a nonnegative supersolution $\bar{u}(t)$ exists, then the solution $u(t)$ obtained in this lemma satisfies $0 \leq u(t) \leq$ $\bar{u}(t)$.

Proof. This lemma is well known. See [10, Theorem 2.1] for details. However, we briefly show the proof for readers' convenience.

If (1.1) has an integral solution, then the solution is also a supersolution. Thus, it is enough to show that (1.1) has an integral solution if (1.1) has a supersolution. Let $\bar{u}$ be a supersolution for $0<t<T$. Let $u_{1}=S(t) \phi$. We define $u_{n}, n=2,3, \ldots$, by

$$
u_{n}=\mathcal{F}\left[u_{n-1}\right] \text {. }
$$

Then we can show by induction that

$$
0 \leq u_{1} \leq u_{2} \leq \cdots \leq u_{n} \leq \cdots \leq \bar{u}<\infty \text { a.e. } x \in \mathbb{R}^{N}, 0<t<T .
$$

This indicates that the $\operatorname{limit}_{\lim _{n \rightarrow \infty}} u_{n}(x, t)$ which is denoted by $u(x, t)$ exists for almost all $x \in \mathbb{R}^{N}$ and $0<t<T$. By the monotone convergence theorem we see that

$$
\lim _{n \rightarrow \infty} \mathcal{F}\left[u_{n-1}\right]=\mathcal{F}[u]
$$

and hence $u=\mathcal{F}[u]$. Then, $u$ is an integral solution of (1.1). It is clear that $0 \leq u(t) \leq$ $\bar{u}(t)$.

Baras-Pierre [1] studied necessary conditions for the existence of an integral solution in the case $p>1$. See also [6] for details of necessary conditions including Proposition 2.2, The following proposition is a variant of [1, Proposition 3.2].

Proposition 2.2. Let $N \geq 1$ and $p=1+2 / N$. If $u(t)$ is an nonnegative integral solution, i.e., $u(t)$ satisfies (1.2) with a nonnegative initial function $\phi$ and some $T>0$, then there exists a constant $\gamma_{0}>0$ depending only on $N$ and $p$ such that

$$
\int_{B(\tau)} \phi(x) d x \leq \gamma_{0}|\log \tau|^{-\frac{N}{2}} \text { for all } 0<\tau<T
$$

where $B(\tau):=\left\{x \in \mathbb{R}^{N}|| x \mid<\tau\right\}$.

Lemma 2.3. Let $q \geq 0$ be fixed, and let

$$
X_{q, \rho}:=\left\{\phi \in L^{1}\left(\mathbb{R}^{N}\right)\left|\int_{\mathbb{R}^{N}}\right| \phi \mid[\log (\rho+|\phi|)]^{q} d x<\infty\right\} .
$$

Then, $\phi \in X_{q, \rho}$ for all $\rho>1$ if and only if $\phi \in X_{q, \sigma}$ for some $\sigma>1$. 
Proof. We consider only the case $q>0$. It is enough to show that $\phi \in X_{q, \rho}$ for all $\rho>1$ if $\phi \in X_{q, \sigma}$ for some $\sigma>1$. Let $\rho>1$ be fixed, and let $\xi(s):=\log (\rho+s) /(\log (\sigma+s))$. By L'Hospital's rule we see that $\lim _{s \rightarrow \infty} \xi(s)=\lim _{s \rightarrow \infty}(s+\sigma) /(s+\rho)=1$. Since $\xi(s)$ is bounded on each compact interval in $[0, \infty)$, we see that $\xi(s)$ is bounded in $[0, \infty)$, and hence there is $C>0$ such that $\log (\rho+s) \leq C \log (\sigma+s)$ for $s \geq 0$. This inequality indicates that $\phi \in X_{q, \rho}$ if $\phi \in X_{q, \sigma}$.

Because of Lemma 2.1, we do not care about $\rho>1$ in (2.2). In particular, if $\phi \in X_{q}$, then $\|g(\phi)\|_{1}<\infty$ for every $\rho>1$.

Proposition 2.4. (i) Let $N \geq 1$ and $1 \leq \alpha \leq \beta \leq \infty$. There is $C>0$ such that, for $\phi \in L^{\alpha}\left(\mathbb{R}^{N}\right)$,

$$
\|S(t) \phi\|_{\beta} \leq C t^{-\frac{N}{2}\left(\frac{1}{\alpha}-\frac{1}{\beta}\right)}\|\phi\|_{\alpha} \text { for } t>0 .
$$

(ii) Let $N \geq 1$ and $1 \leq \alpha<\beta \leq \infty$. Then, for each $\phi \in L^{\alpha}\left(\mathbb{R}^{N}\right)$ and $C_{0}>0$, there is $t_{0}=t_{0}\left(C_{0}, \bar{\phi}\right)$ such that

$$
\|S(t) \phi\|_{\beta} \leq C_{0} t^{-\frac{N}{2}\left(\frac{1}{\alpha}-\frac{1}{\beta}\right)} \text { for } 0<t<t_{0} .
$$

For Proposition 2.4 (i) (resp. (ii)), see [9, Proposition 48.4] (resp. [2, Lemma 8]). Note that $C_{0}>0$ in (ii) can be chosen arbitrary small.

We collect various properties of $g$ defined by (1.7).

Lemma 2.5. Let $q>0$ and let $g_{1}(s):=s[\log (\rho+s)]^{-q}$. Then the following hold:

(i) If $\rho>1$, then $g^{\prime}(s)>0$ for $s>0$.

(ii) If $\rho \geq e$, then $g^{\prime \prime}(s)>0$ for $s>0$.

(iii) If $\rho \geq e$, then $g_{1}(s) \leq g^{-1}(s)$ for $s \geq 0$.

(iv) If $\rho>1$, then there is $C_{1}>0$ such that $g^{-1}(s) \leq g_{1}\left(C_{1} s\right)$ for $s \geq 0$.

(v) If $\rho>e^{q /(p-1)}$, then $g^{-1}(s)^{p} / s$ is nondecreasing for $s \geq 0$.

(vi) If $\rho \geq e$, then, for $\phi \in L^{1}\left(\mathbb{R}^{N}\right)$,

$$
S(t) \phi \leq g^{-1}(S(t) g(\phi)) \text { for } t \geq 0 .
$$

Proof. By direct calculation we have

$$
\begin{aligned}
g^{\prime}(s) & =[\log (\rho+s)]^{q-1}\left\{\log (\rho+s)+\frac{q s}{s+\rho}\right\}, \\
g^{\prime \prime}(s) & =\frac{q[\log (s+\rho)]^{q-2}}{(s+\rho)^{2}}[s\{\log (\rho+s)+q-1\}+2 \rho \log (\rho+s)] .
\end{aligned}
$$

Thus, (i) and (ii) hold.

(iii) Since $\rho \geq e$, we have

$$
g\left(g_{1}(s)\right)=\frac{s}{[\log (\rho+s)]^{q}}\left[\log \left(\rho+\frac{s}{[\log (\rho+s)]^{q}}\right)\right]^{q} \leq \frac{s}{[\log (\rho+s)]^{q}}[\log (\rho+s)]^{q}=s
$$

for $s \geq 0$. By (i) we see that $g^{-1}(s)$ exists and it is increasing. By (2.3) we see that $g_{1}(s) \leq g^{-1}(s)$ for $s \geq 0$.

(iv) Let $\xi(s):=\left(g\left(g_{1}(s)\right) / s\right)^{1 / q}=\log \left(\rho+\frac{s}{[\log (\rho+s)]^{q}}\right) /(\log (\rho+s))$. Then, for each compact interval $I \subset[0, \infty)$, there is $c>0$ such that $\xi(s)>c$ for $s \in I$. By L'Hospital's rule we have

$$
\lim _{s \rightarrow \infty} \xi(s)=\lim _{s \rightarrow \infty} \frac{1+\frac{\rho}{s}}{1+\frac{\rho}{s}[\log (\rho+s)]^{q}}\left\{1-\frac{1}{1+\frac{\rho}{s}} \frac{q}{\log (\rho+s)}\right\}=1,
$$


and hence there is $c_{0}>0$ such that $\xi(s) \geq c_{0}$ for $s \geq 0$. Thus, $g^{-1}\left(c_{0}^{q} s\right) \leq g_{1}(s)$ for $s \geq 0$. Then, the conclusion holds.

(v) By (i) we see that $g(\tau)$ is increasing. Let $s:=g(\tau)$. Then, $g^{-1}(s)^{p} / s=\tau^{p-1}[\log (\rho+\tau)]^{-q}$. Since $\rho>e^{q /(p-1)}$, we have

$$
\frac{d}{d \tau} \frac{\tau^{p-1}}{[\log (\rho+\tau)]^{q}}=\frac{\tau^{p-2}}{[\log (\rho+\tau)]^{q+1}}\left\{(p-1) \log (\rho+\tau)-\frac{q \tau}{\rho+\tau}\right\}>0 .
$$

Thus, $g^{-1}(s)^{p} / s$ is increasing for $s \geq 0$.

(vi) Because of (ii), $g$ is convex. By Jensen's inequality we see that $g(S(t) \phi) \leq S(t) g(\phi)$. Since $g^{-1}$ exists and $g^{-1}$ is increasing, the conclusion holds. The proof is complete.

\section{Existence}

Lemma 3.1. Let $N \geq 1$ and $p=1+2 / N$. Assume that $\phi \geq 0$. If $\phi \in X_{q}$ for some $q \geq N / 2$, then (1.1) has a local-in-time nonnegative mild solution $u(t)$, and $\|u(t)\|_{\infty} \leq$ $C t^{-N / 2}(-\log t)^{-q}$ for small $t>0$.

Proof. First, we consider the case $q=N / 2$. Let $\rho \geq \max \left\{e^{q /(p-1)}, e\right\}$ be fixed. Let $g$ be defined by (1.7). Here, $q=N / 2$ and $g$ satisfies Lemma 2.5. We define

$$
\bar{u}(t):=2 g^{-1}(S(t) g(\phi))
$$

We show that $\bar{u}$ is a supersolution. By Lemma 2.5 (vi) we have

$$
S(t) \phi \leq g^{-1}(S(t) g(\phi))=\frac{\bar{u}(t)}{2} .
$$

Next, we have

$$
\begin{aligned}
\int_{0}^{t} S(t-s) f(\bar{u}(s)) d s & =2^{p} \int_{0}^{t} S(t-s)\left[S(s) g(\phi) \frac{g^{-1}(S(s) g(\phi))^{p}}{S(s) g(\phi)}\right] d s \\
& \leq 2^{p} S(t) g(\phi) \int_{0}^{t}\left\|\frac{g^{-1}(S(s) g(\phi))^{p}}{S(s) g(\phi)}\right\|_{\infty} d s \\
& \leq 2^{p} g^{-1}(S(t) g(\phi))\left\|\frac{S(t) g(\phi)}{g^{-1}(S(t) g(\phi))}\right\|_{\infty} \int_{0}^{t}\left\|\frac{g^{-1}(S(s) g(\phi))^{p}}{S(s) g(\phi)}\right\|_{\infty} d s .
\end{aligned}
$$

Since $g(\phi) \in L^{1}\left(\mathbb{R}^{N}\right)$, by Proposition 2.4 (ii) we have

$$
\|S(t) g(\phi)\|_{\infty} \leq C_{0} t^{-N / 2}
$$

By Lemma 2.5 (v) we see that $g^{-1}(u)^{p} / u$ is nondecreasing for $u \geq 0$. Using (3.3) and Lemma 2.5 (iv), we have

$$
\begin{aligned}
\left\|\frac{g^{-1}(S(s) g(\phi))^{p}}{S(s) g(\phi)}\right\|_{\infty} \leq & \frac{g^{-1}\left(\|S(s) g(\phi)\|_{\infty}\right)^{p}}{\|S(s) g(\phi)\|_{\infty}} \\
& \leq \frac{g^{-1}\left(C_{0} s^{-N / 2}\right)^{p}}{C_{0} s^{-N / 2}} \leq \frac{C_{1}^{p} C_{0}^{2 / N}}{s\left[\log \left(\rho+C_{0} C_{1} s^{-N / 2}\right)\right]^{p q}} \leq \frac{C_{0}^{2 / N} C_{1}^{\prime}}{s(-\log s)^{p q}}
\end{aligned}
$$


for $0<s<s_{0}\left(C_{0}\right)$, where $C_{1}^{\prime}$ is a constant independent of $C_{0}$. Using Lemma 2.5 (iii) and (3.3), we have

$$
\begin{aligned}
\left\|\frac{S(t) g(\phi)}{g^{-1}(S(t) g(\phi))}\right\|_{\infty} & \leq\left\|\frac{S(t) g(\phi)}{g_{1}(S(t) g(\phi))}\right\|_{\infty}=\left\|[\log (\rho+S(t) g(\phi))]^{q}\right\|_{\infty} \\
& \leq\left[\log \left(\rho+\|S(t) g(\phi)\|_{\infty}\right)\right]^{q} \leq\left[\log \left(\rho+C_{0} t^{-N / 2}\right)\right]^{q} \leq C_{2}^{\prime}(-\log t)^{q}
\end{aligned}
$$

for $0<t<t_{0}\left(C_{0}\right)$, where $g_{1}$ is defined in Lemma 2.5 and $C_{2}^{\prime}$ is a constant independent of $C_{0}$. By (3.4) and (3.5) we have

$$
\begin{array}{r}
\left\|\frac{S(t) g(\phi)}{g^{-1}(S(t) g(\phi))}\right\|_{\infty} \int_{0}^{t}\left\|\frac{g^{-1}(S(s) g(\phi))^{p}}{S(s) g(\phi)}\right\|_{\infty} d s \leq C_{0}^{2 / N} C_{1}^{\prime} C_{2}^{\prime}(-\log t)^{q} \int_{0}^{t} \frac{d s}{s(-\log s)^{p q}} \\
=C_{0}^{2 / N} C_{1}^{\prime} C_{2}^{\prime}(-\log t)^{q} \frac{2}{N(-\log t)^{q}}=C_{0}^{2 / N} C_{1}^{\prime} C_{2}^{\prime} \frac{2}{N}
\end{array}
$$

for $0<t<\min \left\{s_{0}\left(C_{0}\right), t_{0}\left(C_{0}\right)\right\}$. By Proposition 2.4 (ii) we can take $C_{0}>0$ such that $2^{p+1} C_{0}^{2 / N} C_{1}^{\prime} C_{2}^{\prime} / N<1$. By (3.1), (3.2) and (3.6) we have

$$
\mathcal{F}[\bar{u}](t)=S(t) \phi+\int_{0}^{t} S(t-s) f(\bar{u}(s)) d s \leq \frac{1}{2} \bar{u}(t)+\frac{1}{2} \bar{u}(t)=\bar{u}(t)
$$

for small $t>0$. Thus, there is $T>0$ such that $\mathcal{F}[\bar{u}] \leq \bar{u}$ for $0<t<T$, and hence $\bar{u}$ is a supersolution. By Lemma 2.1 we see that there is $T>0$ such that (1.1) has a solution for $0<t<T$, and $u(t)$ is clearly nonnegative. Moreover,

$$
0 \leq u(t) \leq \bar{u}(t)=2 g^{-1}(S(t) g(\phi)) \leq C t^{-\frac{N}{2}}(-\log t)^{-q},
$$

which is the estimate in the assertion. We show that $u(t) \in C\left([0, T), L^{1}\left(\mathbb{R}^{N}\right)\right)$. Since $\left\|g^{-1}(u)\right\|_{1} \leq C\|u\|_{1}$, by (3.6) and Proposition 2.4 (i) we have

$$
\begin{aligned}
\|u(t)-S(t) \phi\|_{1} \leq \| \int_{0}^{t} S(t & -s) f(\bar{u}(s)) d s\left\|_{1} \leq C_{0}^{2 / N} C_{1}^{\prime} C_{2}^{\prime} \frac{2}{N}\right\| g^{-1}(S(t) g(\phi)) \|_{1} \\
\leq & C_{0}^{2 / N} C_{1}^{\prime} C_{2}^{\prime} \frac{2}{N} C\|S(t) g(\phi)\|_{1} \leq C_{0}^{2 / N} C_{1}^{\prime} C_{2}^{\prime} \frac{2}{N} C^{\prime}\|g(\phi)\|_{1}
\end{aligned}
$$

for small $t>0$, where $C^{\prime}$ is independent of $C_{0}$. By Proposition 2.4 (ii) we can take $C_{0}>0$ arbitrary small, and hence

$$
\|u(t)-S(t) \phi\|_{1} \rightarrow 0 \text { as } t \downarrow 0 .
$$

Since $S(t)$ is a strongly continuous semigroup on $L^{1}\left(\mathbb{R}^{N}\right)$ (see e.g., 9 , Section 48.2]), we have

$$
\|u(t)-\phi\|_{1} \leq\|u(t)-S(t) \phi\|_{1}+\|S(t) \phi-\phi\|_{1} \rightarrow 0 \text { as } t \downarrow 0 .
$$

It follows from (3.2) and (3.6) that $\left\|\int_{0}^{t} S(t-s) f(\bar{u}(s)) d s\right\|_{1}<\infty$ for $0<t<T$. We see that if $0<t<T$, then

$$
\|u(t+h)-u(t)\|_{1} \rightarrow 0 \text { as } h \rightarrow 0 .
$$

By (3.9) and (3.10) we see that $u(t) \in C\left([0, T), L^{1}\left(\mathbb{R}^{N}\right)\right)$. The proof of (i) is complete. 
Next, we consider the case $q>N / 2$. The argument is the same until (3.6). We have

$$
\begin{array}{r}
\left\|\frac{S(t) g(\phi)}{g^{-1}(S(t) g(\phi))}\right\|_{\infty} \int_{0}^{t}\left\|\frac{g^{-1}(S(s) g(\phi))^{p}}{S(s) g(\phi)}\right\|_{\infty} d s \leq C_{0}^{2 / N} C_{1}^{\prime} C_{2}^{\prime}(-\log t)^{q} \int_{0}^{t} \frac{d s}{s(-\log s)^{p q}} \\
=\frac{C_{1}^{2 / N} C_{1}^{\prime} C_{2}^{\prime}}{p q-1}(-\log t)^{1-\frac{2 q}{N}}
\end{array}
$$

instead of (3.6). Since the RHS of (3.11) goes to 0 as $t \downarrow 0$, the rest of the proof is almost the same with obvious modifications. In particular, (3.7) holds even for $q>N / 2$. We omit the details.

We consider (1.6), where $\phi$ is given in (1.1). By Lemma 3.1 we see that (1.6) has a localin-time solution which is denoted by $w(t)$. We consider the sequence $\left(u_{n}\right)_{n=0}^{\infty}$ defined by (1.8). Then, the following lemma says that $\left\|u_{n}(t)\right\|_{\infty}$ can be controlled by $w(t)$.

Lemma 3.2. Let $u_{n}$ be as defined by (1.8), and let $w$ be a solution of (1.6) on $(0, T)$. Then,

$$
-w(t) \leq u_{n}(t) \leq w(t) \text { for a.e. } x \in \mathbb{R}^{N} \text { and } 0<t<T \text {. }
$$

Proof. It is clear from the definitions of $u_{0}$ and $w(t)$ that

$$
u_{0}(t) \leq w(t) \text { for } 0<t<T
$$

We assume that $u_{n-1}(t) \leq w(t)$ on $(0, T)$. Then, we have

$$
w(t)=S(t)|\phi|+\int_{0}^{t} S(t-s) f(w(s)) d s \geq S(t) \phi+\int_{0}^{t} S(t-s) f\left(u_{n-1}(s)\right) d s=u_{n}(t),
$$

and hence $u_{n}(t) \leq w(t)$ for $0<t<T$. Thus, by induction we see that, for $n \geq 0$,

$$
u_{n}(t) \leq w(t) \text { on } 0<t<T \text {. }
$$

It is clear that $u_{0}(t) \geq-w(t)$ for $0<t<T$. We assume that $u_{n-1}(t) \geq-w(t)$ on $(0, T)$. Then, we have

$$
u_{n}(t)=S(t) \phi+\int_{0}^{t} S(t-s) f\left(u_{n-1}(s)\right) d s \geq-S(t)|\phi|+\int_{0}^{t} S(t-s) f(-w(s)) d s=-w(t),
$$

and hence, $u_{n}(t) \geq-w(t)$ on $(0, T)$. Thus, by induction we see that for $n \geq 0$,

$$
-w(t) \leq u_{n}(t) \text { on } 0<t<T \text {. }
$$

By (3.13) and (3.14) we see that (3.12) holds.

Proof of Theorem 1.3. (i) Let $\left(u_{n}\right)_{n=0}^{\infty}$ be defined by (1.8). Using an induction argument with a parabolic regularity theorem, we can show that, for each $n \geq 1, u_{n} \in C^{2,1}\left(\mathbb{R}^{N} \times(0, T)\right)$ and $u_{n}$ satisfies the equation

$$
\partial_{t} u_{n}=\Delta u_{n}+f\left(u_{n-1}\right) \text { in } \mathbb{R}^{N} \times(0, T)
$$

in the classical sense. Let $K$ be an arbitrary compact subset in $\mathbb{R}^{N} \times(0, T)$, and let $K_{1}$, $K_{2}$ be two compact sets such that $K \subset K_{1} \subset K_{2} \subset \mathbb{R}^{N} \times(0, T)$. Because of Lemma 3.2, $f\left(u_{n-1}\right)$ is bounded in $C\left(K_{2}\right)$. By a parabolic regularity theorem we see that $u_{n}$ is bounded in $C^{\gamma, \gamma / 2}\left(K_{1}\right)$. Using a parabolic regularity theorem again, we see that $u_{n+1}$ is bounded in $C^{2+\gamma, 1+\gamma / 2}(K)$.

In the following we use a diagonal argument to obtain a convergent subsequence in $\mathbb{R}^{N} \times$ $(0, T)$. Let $Q_{j}:=\overline{\left\{x \in \mathbb{R}^{N}|| x \mid \leq j\right\}} \times\left[\frac{T}{j+2}, \frac{(j+1) T}{j+2}\right]$. Since $\left(u_{n}\right)_{n=3}^{\infty}$ is bounded in $C^{2,1}\left(Q_{1}\right)$, 
by Ascoli-Arzerà theorem there is a subsequence $\left(u_{1, k}\right) \subset\left(u_{n}\right)$ and $u_{1}^{*} \in C\left(Q_{1}\right)$ such that $u_{1, k} \rightarrow u_{1}^{*}$ in $C\left(Q_{1}\right)$ as $k \rightarrow \infty$. Since $\left(u_{1, k}\right)_{k=1}^{\infty}$ is bounded in $C^{2,1}\left(Q_{2}\right)$, there is a subsequence $\left(u_{2, k}\right) \subset\left(u_{1, n}\right)$ and $u_{2}^{*} \in C\left(Q_{2}\right)$ such that $u_{2, k} \rightarrow u_{2}^{*}$ in $C\left(Q_{2}\right)$ as $k \rightarrow \infty$. Repeating this argument, we have a double sequence $\left(u_{j, k}\right)$ and a sequence $\left(u_{j}^{*}\right)$ such that, for each $j \geq 1$, $u_{j, k} \rightarrow u_{j}^{*}$ in $C\left(Q_{j}\right)$ as $k \rightarrow \infty$. We still denote $u_{n, n}$ by $u_{n}$, i.e., $u_{n}:=u_{n, n}$. It is clear that $u_{j_{1}}^{*} \equiv u_{j_{2}}^{*}$ in $Q_{j_{1}}$ if $j_{1} \leq j_{2}$. Since $\mathbb{R}^{N} \times(0, T)=\bigcup_{j=1}^{\infty} Q_{j}$, there is $u^{*} \in C\left(\mathbb{R}^{N} \times(0, T)\right)$ such that $u_{n} \rightarrow u^{*}$ in $C(K)$ as $n \rightarrow \infty$ for every compact set $K \subset \mathbb{R}^{N} \times(0, T)$. In particular,

$$
u_{n} \rightarrow u^{*} \text { a.e. in } \mathbb{R}^{N} \times(0, T) .
$$

Let $w$ be a solution of (1.6). It follows from Lemma 3.2 that $\left|u_{n}(x, t)\right| \leq w(x, t)$. Since

$$
\left|G_{t}(x-y) u_{n}(y, t)\right| \leq\left|G_{t}(x-y) w(y, t)\right| \text { for } y \in \mathbb{R}^{N}
$$

and

$$
G_{t}(x-y) w(y, t) \in L_{y}^{1}\left(\mathbb{R}^{N}\right)
$$

by the dominated convergence theorem we see that

$$
\lim _{n \rightarrow \infty} S(t) u_{n}=\lim _{n \rightarrow \infty} \int_{\mathbb{R}^{N}} G_{t}(s-y) u_{n}(y, t) d y=\int_{\mathbb{R}^{N}} G_{t}(s-y) u^{*}(y, t) d y=S(t) u^{*} .
$$

By (3.2) and (3.6) we see that if $T>0$ is small, then

$$
\int_{0}^{t} \int_{\mathbb{R}^{N}} G_{t-s}(x-y) f(w(y, s)) d y d s \leq C g^{-1}(S(t) g(\phi))<\infty
$$

for each $(x, t) \in \mathbb{R}^{N} \times(0, T)$, and hence $G_{t-s}(x-y) f(w(y, s)) \in L_{(y, s)}^{1}\left(\mathbb{R}^{N} \times(0, T)\right)$. Since

$$
\left|G_{t-s}(x-y) f\left(u_{n-1}(y, s)\right)\right| \leq\left|G_{t-s}(x-y) f(w(y, s))\right| \text { for a.e. }(y, s) \in \mathbb{R}^{N} \times(0, T)
$$

and

$$
G_{t-s}(x-y) f(w(y, s)) \in L_{(y, s)}^{1}\left(\mathbb{R}^{N} \times(0, T)\right),
$$

by the dominated convergence theorem we see that

$$
\begin{aligned}
& \lim _{n \rightarrow \infty} \int_{0}^{t} S(t-s) f\left(u_{n-1}(s)\right) d s=\lim _{n \rightarrow \infty} \int_{0}^{t} \int_{\mathbb{R}^{N}} G_{t-s}(x-y) f\left(u_{n-1}(y, s)\right) d y d s \\
&=\int_{0}^{t} \int_{\mathbb{R}^{N}} G_{t-s}(x-y) f\left(u^{*}(y, s)\right) d y d s=\int_{0}^{t} S(t-s) f\left(u^{*}(s)\right) d s .
\end{aligned}
$$

Thus, we take a limit of $u_{n}=\mathcal{F}\left[u_{n-1}\right]$. By (3.15), (3.16) and (3.17) we see that $u^{*}(t)=$ $\mathcal{F}\left[u^{*}\right](t)$ for $0<t<T$.

Since $\left|u_{n}\right| \leq w$, we see that $\left|u^{*}\right| \leq w$. Since $\left|u^{*}\right| \leq w$ in $\mathbb{R}^{N} \times(0, T)$, by (3.8) and the arbitrariness of $C_{0}>0$ we have

$$
\left\|u^{*}(t)-S(t) \phi\right\|_{1}=\left\|\int_{0}^{t} S(t-s) f\left(u^{*}(s)\right) d s\right\|_{1} \leq\left\|\int_{0}^{t} S(t-s) f(w(s)) d s\right\|_{1} \rightarrow 0 \text { as } t \downarrow 0 .
$$

Then, $\left\|u^{*}(t)-\phi\right\|_{1} \leq\left\|u^{*}(t)-S(t) \phi\right\|_{1}+\|S(t) \phi-\phi\|_{1} \rightarrow 0$ as $t \downarrow 0$. Since $\left\|\int_{0}^{t} S(t-s) f(w(s))\right\|_{1}<$ $\infty$ for $0<t<T$, we can show by a similar way to the proof of Lemma 3.1 that $u^{*}(t) \in$ $C\left((0, T), L^{1}\left(\mathbb{R}^{N}\right)\right)$. Thus, $u^{*}(t) \in C\left([0, T), L^{1}\left(\mathbb{R}^{N}\right)\right)$, and hence $u^{*}(t)$ is a mild solution. Since $\left|u^{*}(t)\right| \leq w(t)$, by Lemma 3.1 we have (1.3). The proof of (i) is complete. 


\section{Nonexistence}

Let $0 \leq q<N / 2$ be fixed. Then there is $0<\varepsilon<N / 2-q$. We define $\phi_{0}$ by

$$
\phi_{0}(x):= \begin{cases}|x|^{-N}(-\log |x|)^{-\frac{N}{2}-1+\varepsilon} & \text { if }|x|<1 / e, \\ 0 & \text { if }|x| \geq 1 / e .\end{cases}
$$

Lemma 4.1. Let $0 \leq q<N / 2$, and let $\phi_{0}$ be defined by (4.1). Then the following hold:

(i) $\phi_{0} \in X_{q}\left(\subset L^{1}\left(\mathbb{R}^{N}\right)\right)$.

(ii) The function $\phi_{0}$ does not satisfy (2.1) for any $T>0$.

Proof. (i) We write $\phi_{0}(r)=r^{-N}(-\log r)^{-N / 2-1+\varepsilon}$ for $0<r<1 / e$. Since $\log (e+s) \leq 1+\log s$ for $s \geq 0$, we have

$$
\log \left(e+\left|\phi_{0}\right|\right) \leq 1-N \log r-\left(\frac{N}{2}+1-\varepsilon\right) \log (-\log r) \leq-2 N \log r
$$

for $0<r<1 / e$. Let $B(\tau):=\left\{x \in \mathbb{R}^{N}|| x \mid<\tau\right\}$. Using (4.2), we have

$$
\begin{aligned}
\int_{B(1 / e)}\left|\phi_{0}\right|\left[\log \left(e+\left|\phi_{0}\right|\right)\right]^{q} d x \leq \omega_{N-1} \int_{0}^{1 / e} \frac{(2 N)^{q}(-\log r)^{q} r^{N-1} d r}{r^{N}(-\log r)^{N / 2+1-\varepsilon}} \\
\leq(2 N)^{q} \omega_{N-1} \int_{0}^{1 / e} \frac{d r}{r(-\log r)^{N / 2+1-q-\varepsilon}}=\frac{(2 N)^{q} \omega_{N-1}}{\frac{N}{2}-q-\varepsilon}<\infty,
\end{aligned}
$$

where $\omega_{N-1}$ denotes the area of the unit sphere $\mathbb{S}^{N-1}$ in $\mathbb{R}^{N}$. By (4.3) we see that $\phi_{0} \in X_{q}$. (ii) Suppose the contrary, i.e., there exists $\gamma_{0}>0$ such that (2.1) holds. When $0<\tau<1 / e$, we have

$$
\int_{B(\tau)} \phi_{0}(x) d x=\omega_{N-1} \int_{0}^{\tau} \frac{d r}{r(-\log r)^{N / 2+1-\varepsilon}}=\frac{C}{(-\log \tau)^{N / 2-\varepsilon}},
$$

where $C>0$ is independent of $\tau$. Then,

$$
\gamma_{0} \geq \frac{\int_{B(\tau)} \phi_{0}(x) d x}{(-\log \tau)^{-N / 2}} \geq C(-\log \tau)^{\varepsilon} \rightarrow \infty \text { as } \tau \downarrow 0 .
$$

which is a contradiction. Thus, the conclusion holds.

Proof of Theorem 1.3 (ii). Let $0 \leq q<N / 2$. It follows from Lemma 4.1 (i) that $\phi_{0} \in X_{q}$. By Lemma 4.1 (ii) we see that there does not exist $\gamma_{0}>0$ such that (2.1) holds. By Proposition 2.2 the problem (1.1) with $\phi_{0}$ has no nonnegative integral solution.

\section{UNIQUENESS}

Proof of Theorem 1.5. Let $q>N / 2$. Suppose that (1.1) has two integral solutions $u(t)$ and $v(t)$. Using Young's inequality and the inequality $\|u(t)\|_{\infty} \leq C t^{-N / 2}(-\log t)^{-q}$, we have

$$
\begin{aligned}
\|u(t)-v(t)\|_{1} & \leq \int_{0}^{t}\left\|G_{t-s} *\left\{\left(p|u|^{p-1}+p|v|^{p-1}\right)(u-v)\right\}\right\|_{1} d s \\
& \leq p \int_{0}^{t}\left\|G_{t-s}\right\|_{1}\left(\|u\|_{\infty}^{p-1}+\|v\|_{\infty}^{p-1}\right) d s \sup _{0 \leq s \leq t}\|u(s)-v(s)\|_{1} \\
& \leq C \int_{0}^{t} \frac{d s}{\left\{s^{N / 2}(-\log s)^{q}\right\}^{p-1}} \sup _{0 \leq s \leq t}\|u(s)-v(s)\|_{1} .
\end{aligned}
$$


Since

$$
\int_{0}^{t} s^{-N(p-1) / 2}(-\log s)^{-(p-1) q} d s=\frac{N(-\log t)^{1-2 q / N}}{2 q-N}
$$

and $1-2 q / N<0$, we can choose $T>0$ such that $C \int_{0}^{t} s^{-N(p-1) / 2}(-\log s)^{-(p-1) q} d s<1 / 2$ for every $0 \leq t \leq T$. Then, we have

$$
\sup _{0 \leq t \leq T}\|u(t)-v(t)\|_{1} \leq \frac{1}{2} \sup _{0 \leq s \leq T}\|u(s)-v(s)\|_{1}
$$

which implies the uniqueness.

\section{Acknowledgements}

The author was supported by JSPS KAKENHI Grant Number $19 H 01797$.

\section{REFERENCES}

[1] P. Baras and M. Pierre, Critère d'existence de solutions positives pour des équations semi-linéaires non monotones, Ann. Inst. H. Poincaré Anal. Non Linéaire 2 (1985), 185-212.

[2] H. Brezis and T. Cazenave, A nonlinear heat equation with singular initial data, J. Anal. Math. 68 (1996), 277-304.

[3] C. Celik and Z. Zhou, No local $L^{1}$ solution for a nonlinear heat equation, Comm. Partial Differential Equations 28 (2003), 1807-1831.

[4] H. Fujita, On the blowing up of solutions of the Cauchy problem for $u_{t}=\Delta u+u^{1+\alpha}$, J. Fac. Sci. Univ. Tokyo Sect. I 13 (1966), 109-124.

[5] A. Haraux and F. Weissler, Nonuniqueness for a semilinear initial value problem, Indiana Univ. Math. J. 31 (1982), 167-189.

[6] K. Hisa and K. Ishige, Existence of solutions for a fractional semilinear parabolic equation with singular initial data, Nonlinear Anal. 175 (2018), 108-132.

[7] R. Laister, J. Robinson, M. Sierżȩga and A. Vidal-López, A complete characterisation of local existence for semilinear heat equations in Lebesgue spaces, Ann. Inst. H. Poincaré Anal. Non Linéaire 33 (2016), $1519-1538$.

[8] W. Ni and P. Sacks, Singular behavior in nonlinear parabolic equations, Trans. Amer. Math. Soc. 287 (1985), 657-671.

[9] P. Quittner and P. Souplet, Superlinear parabolic problems. Blow-up, global existence and steady states, Birkhäuser Advanced Texts: Basler Lehrbücher. Birkhäuser Verlag, Basel, 200\%. xii+584 pp. ISBN: 9783-7643-8441-8.

[10] J. Robinson and M. Sierżȩga, Supersolutions for a class of semilinear heat equations, Rev. Mat. Complut. 26 (2013), 341-360.

[11] J. Takahashi, Solvability of a semilinear parabolic equation with measures as initial data, Geometric properties for parabolic and elliptic PDE's, Springer Proc. Math. Stat., vol. 176, Springer, 2016, ISBN: 978-3-319-41538-3, pp. 257-276.

[12] E. Terraneo, Non-uniqueness for a critical non-linear heat equation, Comm. Partial Differential Equations 27 (2002), 185-218.

[13] F. Weissler, Local existence and nonexistence for semilinear parabolic equations in $L^{p}$, Indiana Univ. Math. J. 29 (1980), 79-102.

[14] F. Weissler, Existence and nonexistence of global solutions for a semilinear heat equation, Israel J. Math. 38 (1981), 29-40.

Graduate School of Mathematical Sciences, The University of Tokyo, 3-8-1 Komaba MEguro-Ku TOKYO 153-8914, JAPAN

E-mail address: miyamoto@ms.u-tokyo.ac.jp 